\title{
Avaliação do conhecimento dos anestesiologistas sobre cuidados paliativos
}

Maria de Fátima Oliveira dos Santos ${ }^{1}$, Natália Oliva Teles ${ }^{2}$, Harison José de Oliveira ${ }^{3}$, Nicole de Castro Gomes ${ }^{4}$, Joana Cariri Valkasser Tavares ${ }^{5}$, Edilza Câmara Nóbrega ${ }^{6}$

\section{Resumo}

Avaliar o conhecimento dos cuidados paliativos entre anestesiologistas por meio de questionário individual. A amostra teve 95 profissionais, dos quais 65 do sexo masculino e 30 do feminino. Sessenta e dois anestesiologistas informam que "qualidade de vida" é o termo que melhor expressa os cuidados paliativos e 53 consideram a combinação da assistência casa/hospital a mais conveniente para o atendimento do paciente que requer esses cuidados. Observou-se que $83,2 \%$ dos pesquisados $(n=79)$ não receberam preparação para lidar com paciente que requer cuidados paliativos e $88,4 \%$ comentam com colegas quando um paciente morre $(n=84)$. A maioria dos entrevistados $(n=46)$ discorda da prática de eutanásia. Quanto à autopercepção do conhecimento sobre cuidados paliativos, numa escala de 0 (nenhum conhecimento) a 10 (conhecimento total), a maioria das respostas alcançou média $5(n=28)$, o que mostra a necessidade da reflexão acerca do cuidar na área da anestesiologia.

Palavras-chave: Cuidados paliativos. Assistência terminal. Doente terminal.

\section{Resumen}

\section{La evaluación del conocimiento de los anestesiólogos acerca de los cuidados paliativos}

Evaluar el conocimiento de los cuidados paliativos entre los anestesiólogos a través de un cuestionario individual. La muestra fue de 95, de los cuales 65 eran del sexo masculino y 30 del femenino. Sesenta y dos de los anestesiólogos informan "la calidad de vida" como el término que mejor expresa los cuidados paliativos y 53 consideran que la combinación de cuidado en el hogar / hospital es más conveniente para el cuidado del paciente que lo requiere. Se observó que el $83,2 \%$ de los investigadores $(n=79)$ no recibieron la preparación para lidiar con el paciente que requiere cuidados paliativos y el $88,4 \%$ comentan con sus compañeros cuando un paciente muere $(n=84)$. La mayoría de los encuestados $(n=46)$ no estaban de acuerdo con la práctica de la eutanasia. En cuanto a la autopercepción del conocimiento sobre los cuidados paliativos, en una escala de 0 (ningún conocimiento) a 10 (pleno conocimiento), la mayoría de las respuestas alcanzó el promedio de 5 ( $n=28$ ). Esto demuestra la necesidad de reflexión acerca del cuidar en el área de anestesiología.

Palabras-clave: Cuidado paliativos. Cuidado terminal. Enfermo terminal.

\section{Abstract \\ Evaluation of knowledge among anesthesiologists about palliative care}

To assess anesthesiologists' knowledge about palliative care through an individual questionnaire. The sample was 95, with 65 male and 30 female. Sixty-two of the anesthesiologists reported quality of life as the term that best expresses the palliative care, and 53 consider the combination of home care/ hospital the most convenient for the treatment of a patient who requires palliative care. It was observed that $83.2 \%$ of the interviewed $(n=79)$ have not been prepared for patients who require palliative care and that $88.4 \%$ mention to a colleague when a patient dies $(n=84)$. Most respondents $(n=46)$ disagreed with the practice of euthanasia. As for the perception of palliative care on a scale of 0 (no knowledge) to 10 (full knowledge), there was a majority of the average response of $5(n=28)$. It shows the need to reflect about care in the filed of anesthesiology. Key words: Paliative care. Terminal care. Terminally ill.

\section{Aprovação CEP Hospital Universitário Lauro Wanderley/UFPB - 359/09}

1. Doutoranda fatimadeosantos@hotmail.com - Faculdade de Medicina Nova Esperança, João Pessoa/PB, Brasil 2. Doutora natalia. oteles1@gmail.com - Faculdade de Medicina da Universidade do Porto, Portugal 3. Especialista harisonoliveira@bol.com.br - Hospital Santa Isabel, João Pessoa/PB, Brasil 4. Graduanda nicoledecastrog@gmail.com 5. Graduanda joanavalkasser@gmail.com 6. Graduanda edxinobrega@hotmail.com - Universidade Federal da Paraíba, João Pessoa/PB, Brasil. 
Cuidados paliativos (CP) são um modelo de cuidar que só se expressou de forma especializada por volta de 1967, quando Cecily Saunders fundou o St. Christopher Hospice, em Londres, dando início ao trabalho de difundir a filosofia do hospice movement de modo a promover a qualidade de vida ante doenças sem possibilidade de cura. Esses cuidados têm como meta assistir os pacientes mediante abordagem destinada a melhorar a qualidade de vida nas doenças que ameaçam a continuidade da existência, entendendo o processo de morrer como inevitável e natural. Os CP baseiam-se em terapêuticas que buscam oferecer, no momento próprio, qualidade de vida digna e diminuir os sintomas que a doença ocasiona, respeitando sempre os direitos individuais, sem esquecer que a família também deve ser assistida ${ }^{1-2}$.

Os CP são procedimentos que buscam controlar a dor e outros sintomas, como os de ordem psicológica, social e espiritual. Logo, o seu objetivo é proporcionar melhor qualidade de vida aos pacientes e familiares, que enfrentam problemas associados com doenças ameaçadoras ${ }^{3}$. Os cuidados paliativos chegaram ao Brasil no início da década de 80, quando o sistema de saúde priorizava a modalidade hospitalocêntrica, essencialmente curativa.

Segundo Kovács ${ }^{4}$, o conceito de CP mudou ao longo do tempo à medida que a filosofia de cuidado foi se desenvolvendo nos ambientes de saúde. Tradicionalmente, eram vistos como procedimentos aplicáveis apenas no momento da morte, mas atualmente são oferecidos desde o estágio inicial de doença terminal progressiva, avançada, incurável, até os últimos momentos de vida. Ressalte-se que esse cuidado deve valorizar aspectos que ficaram em segundo plano: as dimensões humana e éticoespiritual da pessoa ${ }^{4-6}$.

Os CP traduzem mudança significativa no papel dos profissionais dos serviços de saúde, que além de cuidarem da vida devem também cuidar do processo de morrer, haja vista serem intervenções destinadas àqueles em situação de fim de vida, voltadas a amenizar sintomas desagradáveis, provocados pela doença incurável. Considerando que a formação dos profissionais de saúde sempre esteve voltada para os aspectos biológicos, sendo a sua prática predominantemente individual, consistindo em intervenções fragmentadas de diferentes profissionais para o mesmo paciente, compreende-se que seja tão importante utilizar preceitos humanitários entre os profissionais ${ }^{7,8}$.

Em síntese, os cuidados paliativos estão centrados no direito de o paciente viver os dias que lhe restam e de morrer com dignidade, constituindo campo interdisciplinar de cuidados totais, ativos e integrais dispensados aos pacientes com doenças avançadas e em fase terminal. Esse conjunto de ações interdisciplinares busca oferecer a "boa morte" aos pacientes com doenças terminais, bem como apoio aos familiares e cuidadores ${ }^{9}$.

Especificamente no campo da anestesia, a expansão dos cuidados paliativos é ainda mais recente, verificando-se que os desafios para o anestesista que assiste o paciente que requer cuidados paliativos vêm aumentando devido ao crescente progresso obtido com as novas técnicas de analgesia e sedação. $O$ cuidar em anestesia paliativa destina-se a prover conforto, proporcionar ao outro o seu próprio cuidado e dar-lhe o poder de se responsabilizar por isso. Dentre essas novas técnicas destaca-se a analgesia controlada pelo paciente (ACP) ${ }^{10-11}$.

É, enfim, agir e reagir adequadamente frente a situação de morte com o doente e a família, lutando para preservar sua integridade física, moral, emocional e espiritual, conectando-se com o paciente e comprometendo-se a auxiliá-lo e permitir que também possa decidir por si mesmo como e quando utilizar a sedação paliativa para amenizar seus sintomas ${ }^{10,12}$. Cuidar, em anestesia paliativa, é prover o alívio e reconhecer seu paciente como ser humano único. Por isso, é fundamental o controle de sintomas como a dor e a fadiga, a anorexia, a constipação e a dispneia, entre outros ${ }^{12-13}$. Tendo como base esses pressupostos, o objetivo deste estudo foi avaliar o conhecimento sobre cuidados paliativos entre médicos anestesiologistas da cidade de João Pessoa, na Paraíba, Brasil.

\section{Método}

Foi realizada pesquisa do tipo exploratória, descritiva, com abordagem quantitativa, com anestesiologistas da Sociedade de Anestesiologia do Estado da Paraíba (SAEPB) que exercem suas atividades profissionais na cidade de João Pessoa/PB e aceitaram participar voluntariamente neste trabaIho, nos meses de janeiro e fevereiro de 2012. 
Dos 126 anestesiologistas registrados na SAEPB, contatados por telefone, $95(75,4 \%)$ aceitaram fazer parte da amostra. Os dados foram coletados pessoalmente pelos pesquisadores, mediante questionário estruturado composto por perguntas fechadas do tipo múltipla escolha, que continha questões sobre os cuidados paliativos.

A pesquisa foi elaborada de acordo com as recomendações éticas da Resolução CNS/MS 196/92, substituída pela Resolução CNS/MS 466/12 ${ }^{14}$. Os participantes confirmaram o consentimento por meio da assinatura do termo de consentimento livre e esclarecido (TCLE), do qual receberam cópia. Justifica-se a escolha desses profissionais por muitos deles assistirem de forma contínua ou esporádiaca pacientes nessa situação, que requerem seus cuidados. Entretanto, o estudo não se restringiu àqueles profissionais que lidam exclusivamente com paciente que se encontra com a doença em fase terminal, sendo o critério de inclusão ser anestesiologista com atividade na cidade de João Pessoa.

Para a análise dos dados utilizou-se o programa estatístico Statistical Package of Social Sciences for Windows (SPSS), versão 19.0. Os dados descritivos foram expressos em valores percentuais, média, mediana, moda e desvio padrão. Para realizar as análises dos dados foi utilizado o teste de qui-quadrado com simulação de Monte Carlo.

\section{Resultados e discussão}

O estudo contou com a participação de 95 médicos anestesiologistas da cidade de João Pessoa/PB, sendo 65 do sexo masculino $(68,4 \%)$ e 30 do feminino (31,6\%). Quanto ao estado civil, houve predominância dos casados, com $65,3 \%(n=62)$, sendo a menor parcela da amostra composta por indivíduos viúvos, representando apenas $2,1 \%(n=2)$ do total.

A maior parte dos indivíduos tinha idade entre 41 e 50 anos (26,3\%) e apenas $7,4 \%(n=7)$ entre 25 e 30 anos. Quanto ao tempo de formado, $33,7 \%(n=32)$ possuíam 30 anos ou mais, com média de 21,3 anos. No que se refere à carga de trabalho semanal, a maior parcela $43,2 \%$ ( $n=41)$ dos pesquisados trabalha entre $40 \mathrm{e}$ 70 horas semanais, com média de 65,1 horas. Em relação à confissão religiosa, os maiores grupos da amostra são de católicos $(73,6 \% ; n=70)$ e protestantes $(13,7 \%$; $n=13)$. A maioria $63,2 \%(n=60)$ afirmou ser praticante.
Na Tabela 1 observam-se respostas às perguntas: "Palavra que expressa CP" e "onde atender ao paciente que requer CP". Verificou-se que o termo "qualidade de vida" é o que melhor expressa os cuidados paliativos (65,3\%; $n=62$ ). Quando indagados onde deve ser $\mathrm{o}$ atendimento do paciente que requer cuidados paliativos, a maioria dos entrevistados (55,8\%; n=53) respondeu "casa/hospital", seguido por $36,8 \%(n=35)$ que consideram a "casa" e apenas 3,2\% ( $n=3)$, o "hospice".

Tabela 1. Dados referentes às perguntas: "Palavra que expressa cuidados paliativos e onde atender ao paciente que requer $C P^{\prime \prime}$

\begin{tabular}{|c|c|c|}
\hline Variável & $\mathbf{N}$ & $\%$ \\
\hline \multicolumn{3}{|c|}{ Palavra que expressa cuidados paliativos } \\
\hline Dor & 2 & 2,1 \\
\hline Família & 1 & 1,0 \\
\hline Morte digna & 24 & 25,3 \\
\hline Não respondeu & 2 & 2,1 \\
\hline Ortotanásia & 4 & 4,2 \\
\hline Qualidade de vida & 62 & 65,3 \\
\hline \multicolumn{3}{|c|}{$\begin{array}{l}\text { Onde deve ser } 0 \text { atendimento do paciente que } \\
\text { requer cuidados paliativos }\end{array}$} \\
\hline Casa & 35 & 36,8 \\
\hline Casa/hospital & 53 & 55,8 \\
\hline Hospice & 3 & 3,2 \\
\hline Hospital & 3 & 3,2 \\
\hline Não respondeu & 1 & 1,0 \\
\hline
\end{tabular}

$\mathrm{N}=$ número de anestesiologistas.

A Tabela 2 resume as respostas obtidas às perguntas: "Recebeu preparação para lidar com paciente que requer CP?"; "Vivenciou a experiência de perda pessoal?"; "Quando acontece a morte de paciente que você assistiu, aborda [o fato] com os seus colegas anestesiologistas?". Para a maioria dos pesquisados - 83,2\% ( $n=79)$ - faltou preparação para lidar com pacientes que requerem CP terminais. Quando indagados se vivenciaram a experiência da perda pessoal, 92,6\% $(n=88)$ responderam que sim. Relativamente à pergunta "Quando acontece a morte de um paciente que você assistiu, comenta com seus colegas anestesistas?", 88,4\% ( $n=84)$ responderam afirmativamente. 
Tabela 2. Dados referentes às perguntas: "Recebeu preparação para lidar com paciente que requer cuidados paliativos"; "Vivenciou a experiência de perda pessoal" e "Aborda com os colegas a morte de um paciente"

\begin{tabular}{|c|c|c|}
\hline Perguntas/respostas & $\mathbf{N}$ & $\%$ \\
\hline \multicolumn{3}{|c|}{$\begin{array}{l}\text { Recebeu preparação para lidar com paciente } \\
\text { que requer cuidados paliativos? }\end{array}$} \\
\hline Sim & 16 & 16,8 \\
\hline Não & 79 & 83,2 \\
\hline \multicolumn{3}{|c|}{ Vivenciou a experiência de perda pessoal? } \\
\hline Sim & 88 & 92,6 \\
\hline Não & 7 & 7,4 \\
\hline \multicolumn{3}{|c|}{$\begin{array}{l}\text { Quando acontece a morte de paciente que } \\
\text { você assistiu, aborda [o fato] com seus colegas } \\
\text { anestesiologistas? }\end{array}$} \\
\hline Sim & 84 & 88,4 \\
\hline Não & 11 & 11,6 \\
\hline
\end{tabular}

$\mathrm{N}=$ número de anestesiologistas.

A Tabela 3 resume os dados obtidos quanto ao posicionamento em relação à prática da eutanásia, relativamente ao sexo dos inquiridos. Assim, verificou-se que tanto $29(63,0 \%)$ dos indivíduos inquiridos do sexo masculino como $17(37,0 \%)$ do sexo feminino discordam de sua prática, totalizando 46 indivíduos. $O$ teste do qui-quadrado sugere não existir associação entre os dados. Assim,depreende-se que o sexo não interfere no posicionamento quanto à prática da eutanásia.

Tabela 3. Dados referentes ao posicionamento em relação à prática da eutanásia quanto ao sexo

\begin{tabular}{|c|c|c|c|c|c|c|c|c|c|}
\hline & \multicolumn{2}{|c|}{$\begin{array}{l}\text { Não } \\
\text { sabe }\end{array}$} & \multicolumn{2}{|c|}{$\begin{array}{c}\text { Concor- } \\
\text { da }\end{array}$} & \multicolumn{2}{|c|}{$\begin{array}{c}\text { Discor- } \\
\text { da }\end{array}$} & \multicolumn{2}{|c|}{$\begin{array}{l}\text { Não res- } \\
\text { pondeu }\end{array}$} & \multirow[t]{2}{*}{$\mathbf{N}$} \\
\hline & $\mathrm{N}$ & $\%$ & $N$ & $\%$ & $\mathrm{~N}$ & $\%$ & $N$ & $\%$ & \\
\hline Feminino & 8 & 38,1 & 4 & 14,8 & 17 & 37,0 & 1 & 100,0 & 30 \\
\hline Masculino & 13 & 61,9 & 23 & 85,2 & 29 & 63,0 & 0 & - & 65 \\
\hline Total & 21 & 22,1 & 27 & 28,4 & 46 & 48,5 & 1 & 1,0 & 95 \\
\hline
\end{tabular}

$\mathrm{N}=$ número de anestesiologistas.

Quanto à autopercepção dos anestesiologistas em relação ao conhecimento sobre $\mathrm{CP}$, numa escala de 0 a 10, observou-se que a maior frequência (moda) foi 5; a média foi 5,8; a mediana, 6,0; e o desvio padrão, 1,54 para $n=28$. Evidenciou-se nível de significância de 0,755. Logo, a maior parte dos indivíduos da amostra atribuiu nota que variou entre 4 e 7, para desvio padrão de 1,54. O teste qui-quadrado não sugeriu associação entre os dados, ou seja, o sexo não influenciou na atribuição da nota.
Discutir a temática dos cuidados paliativos pressupõe a realização de exercício reflexivo, que envolve família, cuidador, morte e autonomia do paciente, entre outros. De início, observa-se serem poucas as pesquisas sobre cuidados paliativos direcionadas aos anestesiologistas e pouco se sabe sobre a opinião e o posicionamento desses profissionais. Em relação ao questionamento que procurava identificar a palavra adequada para expressar "cuidados paliativos", 65,3\% dos pesquisados indicaram o termo "qualidade de vida" como o que melhor se presta à definição.

O paciente que se encontra com a doença em fase terminal e em tratamento de quimioterapia apresenta diminuição das funções física, emocional, cognitiva e social, pois nesse momento ocorre o aumento dos sintomas fadiga, náuseas e vômitos, dor, insônia, perda de apetite e diarreia. Por isso, é tão difícil a mensuração de qualidade de vida (QV) do paciente terminal. Ressalte-se que essa definição inclui seis domínios principais: saúde física, estado psicológico, níveis de independência, relacionamento social, características ambientais e padrão espiritual. Portanto, a qualidade de vida é uma noção eminentemente humana, que tem sido entendida como grau de satisfação encontrado na vida familiar, amorosa, social e ambiental, e na própria estética existencial ${ }^{15-16}$.

Aprofundando o assunto, observa-se que o anestesiologista que atua em cuidados paliativos busca amenizar ou diminuir o desconforto por que passam o paciente e família. Por ter o privilégio da proximidade com quem está em processo de morte, pode transformar essa convivência em fonte de conforto para quem arrosta a inevitabilidade da morte. Em face dessa circunstância, os cuidados paliativos buscam oferecer ao paciente e a sua família a meIhor qualidade de vida no tempo que ainda resta àquele ${ }^{15,17}$.

Entre as opções para o atendimento do paciente que requer cuidados paliativos, os anestesiologistas entrevistados reconhecem que a combinação preferida é casa/hospital (55,8\%; $n=53)$. Nesse sentido, até o médico cuidador entende que a combinação casa/hospital é a mais completa. Isto significa alteração em relação ao local habitual de morte e induz a necessidade de criar e implementar programas efetivos e integrados nos hospitais, para prestar esse atendimento, dado que a tendência é de que os pacientes invertam sua preferência quanto ao local de atendimento - o que parece estar acontecendo, manifestando-se no fato de os doentes preferirem, cada vez mais, permanecer em casa, junto da família e das pessoas que amam. 
O Conselho Federal de Medicina editou a Resolução $1.805 / 06$, que todos os médicos precisam conhecer. Trata da terminalidade da vida e tem caráter deontológico, garantindo os cuidados essenciais para aliviar os sintomas que podem levar ao sofrimento, respeitando sempre a vontade do paciente e trazendo-lhe mais conforto no estado terminal. Posta a questão nesses termos, a conduta a ser adotada para o doente em processo de morte inevitável será sempre polêmica, questionando-se se valerá a pena retirar o paciente de casa e transportá-lo para o hospital apenas para morrer ${ }^{18}$.

A maioria dos anestesiologistas pesquisados $(83,2 \% ; n=79)$ declarou não ter recebido preparação para lidar com pacientes que requerem cuidados paliativos. Essas considerações vão ao encontro de estudos realizados em diversos países que mostram lacuna na formação dos profissionais de saúde no tocante aos cuidados paliativos, indicando a necessidade de estimular essa formação ${ }^{19,20}$. Embora os cuidados paliativos sejam pouco divulgados também entre os anestesiologistas, essa abordagem é extremamente relevante para esse grupo profissional que, muitas vezes, é aquele designado a assistir o doente nos últimos momentos de vida.

Em decorrência dessa deficiência na formação profissional, a adoção de medidas fúteis muito provavelmente decorre do desconhecimento dos profissionais sobre os cuidados paliativos. A respeito de tal situação no Brasil, constata-se que existem diversos desafios a vencer, dentre eles o de superar essa possível deficiência no processo de formação dos profissionais de saúde quanto à finitude da vida. É indispensável que o anestesiologista esteja preparado para os cuidados paliativos, valorizando a autonomia do paciente, cujo posicionamento deve respeitar sempre que possível.

Se é viável constatar que a mudança de mentalidade dos profissionais para a adoção dos CP é necessária, percebe-se também que nem sempre estes estão dispostos e receptivos a nova mudança com quebra de paradigmas. Considerando-se que o cuidar do paciente terminal vai além dos procedimentos técnicos, devemos abordar a dimensão humana, englobando - dentro das possibilidades aspirações, desejos, necessidades e vontades do paciente terminal. Ante isso, somos levados a crer que será necessário realizar árduo trabalho, em termos de mudanças (pessoais, humanas e sociais), a partir dos aparelhos formadores que moldam profissionais com excelente preparação técnica e nenhuma ênfase humanística ${ }^{21,22}$.
Como consequência dos avanços da tecnologia, a procura por profissionais tecnicamente habilitados é e será sempre cada vez maior, em detrimento da preocupação com a alma humana. Esse fenômeno do avanço da tecnologia tem levado a medicina a segmentar-se cada vez mais, oferecendo, além das especialidades, as subespecialidades, o que faz com que os seus profissionais se dediquem apenas a alguns segmentos do corpo, levando-os a esquecer a integralidade da pessoa humana como ser dotado de corpo e alma, e que, por isso, necessita de visão holística no tratamento integral da sua saúde ${ }^{22}$.

Os anestesiologistas que atuam como cuidadores encontram-se também em situação de fragilidade quando se deparam com paciente sem possibilidades terapêuticas ${ }^{23}$. Observou-se no presente estudo que muitos deles comentam com os colegas a morte de pacientes sob seus cuidados, vez que costumam compartilhar informações sobre o dia a dia laboral. Tal situação parece ser relativamente comum, considerando-se que esses profissionais têm papel imprescindível no cuidar do paciente que está morrendo, visto que a fase terminal pode ser terrivelmente difícil tanto para o doente quanto para o cuidador, pois o que se pode fazer pelo paciente é muito pouco. Não obstante, referem que os médicos consideram a morte do paciente como falha de sua ciência e, frustrados, encerram sentimentos de culpa por não conseguirem evitá-la ${ }^{24}$. Para Horta, os profissionais de saúde e de outras áreas carecem de reflexão para enfrentar os desafios do processo de morrer e da morte - que pode ajudar, inclusive, na elaboração de suas experiências ${ }^{25}$.

Sobre a eutanásia, os anestesiologistas do estudo posicionaram-se da seguinte maneira: 46 discordam, 27 concordam e 22 não responderam ou não sabem. A eutanásia não é aceita em boa parte das sociedades, sendo conduta ética proibida, constituindo-se em antecipação da morte de alguém em ato de misericórdia sem qualquer lucro pessoal. Na perspectiva de Cabral, a palavra "eutanásia", de origem grega, foi introduzida por Francis Bacon, no Organon. Com o significado etimológico de "boa morte", isto é, suave, pacífica, sem sofrimento, a eutanásia é caracterizada como ato médico cuja finalidade é eliminar a dor e a indignidade da doença terminal, mediante indução direta e ativa da morte de seu portador ${ }^{2,26,27}$.

Evidenciou-se neste trabalho que os conhecimentos sobre cuidados paliativos são insuficientes, o que pode ser explicado como falha na formação médica, período em que pouco se fala da morte. Assim, os anestesiologistas devem receber preparação para saber como lidar com os desafios que surgirão no 
campo do atendimento ao paciente que requer cuidados paliativos. É também necessário que o anestesiologista possua, para além da competência técnicocientífica, competência humana, vivenciando os verdadeiros valores para o agir coerente e responsável, pois neste momento o profissional deve compreender os temores e medos dos pacientes, bem como esclarecer dúvidas sobre os aspectos inerentes ao ato anestésico de forma a minimizar fatores que possam interferir no bem-estar dos mesmos ${ }^{28}$.

Nessa perspectiva, há que se cuidar da formação do médico, por meio da reflexão e discussão em todo o seu processo de formação e aperfeiçoamento. Assim, é importante reconhecer a necessidade de formação no campo específico dos cuidados paliativos, de forma a contribuir para a melhoria da prestação dos cuidados de saúde pelos profissionais de saúde, devido a essas preocupações serem conflitos constantes e diários de qualquer médico que lide com doentes terminais ${ }^{16,29}$.

\section{Considerações finais}

O presente estudo apresentou e analisou o conhecimento dos anestesiologistas da cidade de João Pessoa, na Paraíba, sobre os cuidados paliativos. Este tipo de cuidado se encontra em campo filosófico e conceitual ainda em construção, de modo que sua prática é desafiadora para essa categoria de profissionais médicos, especialmente porque tanto a própria ciência quanto a formação profissional focam na prática tecnicista voltada à cura.
Embora nos cuidados paliativos a ação também seja movida pela proposta de atenção técnicocientífica, incorpora outras questões subjetivas. A assistência médica ao enfermo que necessita dos cuidados paliativos possui - essencialmente - dimensão humana. Para atender cabalmente esses pacientes, requer a adoção de medidas que não visam mais curar, mas sim aliviar o sofrimento; por isso, a razão da necessidade de cuidados paliativos é ter como prioridade o valor da dignidade da pessoa, o que implica a considerar integralmente, não somente do ponto de vista do diagnóstico, mas também em relação ao tratamento da doença e o que pode ser feito quando se esgotam os recursos da terapêutica.

Nesse contexto, evidencia-se a relevância da reflexão sobre os cuidados paliativos para essa categoria de profissionais, ressaltando-se o envolvimento dos anestesiologistas no cuidado desses pacientes nos momentos finais de vida, cientes da importância de seu papel como pilar dos cuidados médicos em face da indispensável assistência que devem prestar-Ihes nos últimos instantes, aliviandoIhes a dor e o sofrimento.

Acredita-se que os dados apresentados nesta pesquisa podem colaborar para evidenciar a necessidade de valorizar o anestesiologista cuidador no processo da assistência ao paciente que precisa de cuidados paliativos. É fundamental inculcarmos a necessidade de futuras pesquisas na área de cuidados paliativos com os anestesiologistas, com vistas a contribuir para a melhoria do desempenho dessa tarefa médica.

\section{Agradecimentos}

\section{Referências}

1. Internacional Association for Hospice and Palliative Care. Manual of palliative care. [Internet]. (acesso 25 jul. 2012). Disponível: http://hospicecare.com/about-iahpc/publications/manual-ofpalliative-care/

2. Silva RCF, Hortale VA. Cuidados paliativos oncológicos: elementos para o debate de diretrizes nesta área. Cad Saúde Pública. 2006;22(10):2.055-66.

3. World Health Organization. Definition of palliative care. [Internet]. (acesso 13 ago. 2012). Disponível: http://tinyurl.com/5228js

4. Kovács MJ. Educação para a morte: desafio na formação de profissionais de saúde e educação. São Paulo: Casa do Psicólogo; 2003. p. 178.

5. Melo AGC. Os cuidados paliativos: uma nova abordagem em evolução no mundo. Arquivos do IV Simpósio Brasileiro sobre Dor - Simbidor. São Paulo; 1999. p. 294-6.

6. Pessini L, Hossne WS. Terminalidade da vida e o novo Código de Ética Médica. Revista Bioethikos. 2010;4(2):127-9.

7. Pessini L. A filosofia dos cuidados paliativos: uma resposta diante da obstinação terapêutica. In: Pessini L, Bertachini L, organizadores. Humanização e cuidados paliativos. $3^{a}$ ed. São Paulo: Loyola; 2006. p. 181-208. 
8. Ayres JRCM. Sujeito, intersubjetividade e práticas de saúde. Ciênc Saúde Coletiva. 2001;6(1):6372.

9. Floriani CA, Schramm FR. Desafios morais e operacionais da inclusão dos cuidados paliativos na rede de atenção básica. Cad Saúde Pública. 2007;23(9):2.072-80.

10. Nogueira FL, Sakata RK. Sedação paliativa do paciente terminal. Rev Bras Anestesiol. 2012;62(4):586-92.

11. Gonçalves JA. Sedation and expertise in palliative care. J Clin Oncol. 2006;24(25):e44-5.

12. Elsayem A, Curry E, Boohene J, Munsell MF, Calderon B, Hung F et al. Use of palliative sedation for intractable symptoms in the palliative care unit of a comprehensive cancer center. Support Care Cancer. 2009;17(1):53-9.

13. Floriani CA, Schramm FR. Palliative care: interfaces, conflicts and necessities. Ciênc Saúde Coletiva. 2008;13(2 Suppl):2.123-32.

14. Brasil. Normas de diretrizes para pesquisa envolvendo seres humanos. Brasília: Ministério da Saúde; 1996. Disponível: http://www.bioetica.ufrgs.br/res19696.htm

15. Sawada NO, Nicolussi AC, Okino L, Cardozo FMC, Zago MMF. Avaliação da qualidade de vida de pacientes com câncer submetidos à quimioterapia. Rev Esc Enferm USP. 2009;43(3):581-7.

16. Benarroz MO, Faillace GBD, Barbosa LA. Bioética e nutrição em cuidados paliativos oncológicos em adultos: [revisão]. Cad Saúde Pública. 2009;25(9):1.875-82.

17. World Health Organization. The World Health Organization quality of life assessment (WHOQOL) position paper from the World Health Organization. Soc Sci Med. 1995;41(10):1.403-9.

18. Conselho Federal de Medicina. Resolução CFM no 1.805, de 28 de novembro de 2006. Na fase terminal de enfermidades graves e incuráveis é permitido ao médico limitar ou suspender procedimentos e tratamentos que prolonguem a vida do doente, garantindo-lhe os cuidados necessários para aliviar os sintomas que levam ao sofrimento, na perspectiva de uma assistência integral, respeitada a vontade do paciente ou de seu representante legal. Diário Oficial da União. 28 nov. 2006;seção I, p. 169.

19. Vial PC, Ibanez PL, Umana AV, Reyes MMD, Viviani P, Nervi FO. Autoevaluación sobre formación en medicina paliativa en una cohorte de residentes. Rev Méd Chile. 2004;132(4):445-52.

20. Vincent JL. Forgoing life support in western European intensive care units: the results of an ethical questionnaire. Crit Care Méd. 1999;27(8):1.626-33.

21. Tapiero AA. Las diferentes formas de morir: reflexiones éticas. An Med Interna. 2004;21(7):355-8.

22. Moritz RD, Machado FO, Heerdt M, Rosso B, Beduschi G. Avaliação das decisões médicas durante o processo do morrer. Rev Bras Ter Intensiva. 2009;21(2):141-7.

23. Ribeiro DC. Autonomia: viver a própria vida e morrer a própria morte. Cad Saúde Pública. 2006;22(8):1.749-54.

24. Sgreccia E. A pessoa humana e seu corpo. Manual de bioética: fundamentos e ética biomédica. $2^{a}$ ed. São Paulo: Loyola; 2002. p. 111-38.

25. Horta AL. Processo de morte e morrer no paciente, na família e nos profissionais de enfermagem. Rev Nursing. 2002;(54):15-7.

26. Cabral RAP. Eutanásia e distanásia: clarificações necessárias. [Internet]. Lisboa: Academia das Ciências de Lisboa; 1990 (acesso 14 jun. 2012). Disponível: http://www.braga.ucp.pt/resources/ documents/FACFIL/cvdocentes/rcabral.html

27. Veiga F, Monteiro F, Teles N, Guerreiro T. Eutanásia: pelo direito de morrer em paz. Cad Bioética. 2000;24:85-113.

28. Smedira NG, Evans BH, Grais LS, Cohen NH, Lo B, Cooke $M$ et al. Withholding and withdrawal of life support from the critically ill. N Engl J Med. 1990;322(5):309-15.

29. Bifulco VA. A morte na formação dos profissionais de saúde. Prática Hospitalar. 2006;45(2):164-6.

\section{Participação dos autores}

Maria de Fátima O. Santos participou da concepção e desenho do estudo por meio da elaboração do projeto de pesquisa e da análise estatística e interpretação dos dados, além da redação do manuscrito. Natália Oliva Teles auxiliou na revisão da literatura e análise dos dados. Harison J. de Oliveira auxiliou na análise estatística e sua interpretação. Nicole de Castro e Joana Cariri contribuíram com a revisão crítica do manuscrito e Edilza Câmara, com a revisão da literatura.

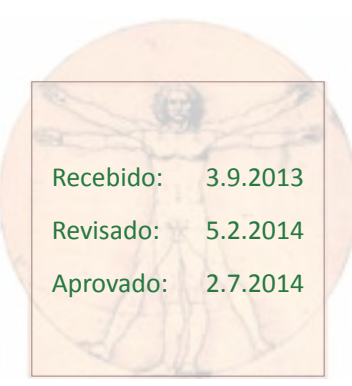

